\title{
ON CONCEPTUALIZATION AS A SYSTEMATIC PROCESS
}

\author{
A.J.J. van Breemen and Janos J. Sarbo \\ Institute for Computing and Information Sciences, Radboud University, Nijmegen, The Netherlands, janos@cs.ru.nl
}

Keywords: conceptualization, signs, Peirce, semiotic sheet

\begin{abstract}
We are concerned with the early phases of ER-modeling consisting in the primary conceptualization of the underlying application domain. To this end we introduce a process model for the generation of a domain description. In virtue of its close relation with cognitive activity, this model enables the modeler as well as the user to comprehend the concepts of the resulting domain in a natural way.
\end{abstract}

\section{Introduction}

Is it possible to classify the concepts 'generated' during modeling processes in types? By knowing the kinds of concepts that can be distinguished, we could make conceptualization more systematic which in turn may improve its effectiveness (less errors) and conceptual efficiency (Liu, 2000). To this end we introduce a process model for conceptualization. Our model can be derived on the basis of an analysis of the properties of cognitive activity, as it has been shown at last year ICEIS conference (Sarbo, 2007); and on the basis of a semiotic analysis of representation, as it will be developed in this paper. Whereas the first approach may provide more insight in the computational aspects of conceptualization as a process, the second one opens the way towards using the types of conceptualization events as pigeonholes during problem specification.

In past research we have shown that our process model enables uniform representation in different knowledge domains. This is why our approach may be interesting for organizations involved in modeling processes. The aim of such processes is to make explicit and reconcile knowledge obtained from different stake holders, in a (semi-)formal model. The knowledge involved is generated with the aim of realizing goals in individual conceptualization processes that themselves serve a common goal: the 'generation' of a shared, common understanding of a prob- lem. In virtue of this common goal, conceptualization in organizations offers an excellent occasion to execute control.

The knowledge involved in each individual business process is tightly knit with the goal it serves to realize. This shapes the attitude of the stake holders of different domains. It is only natural that, as a rule, they will understand and value other processes from their own habitual point of view. Reconciliation starts with the decision to consciously subsume knowledge needed for the different processes under the common goal. But this only expresses the intention. In order to realize the intention, it is necessary to explicate the domain specific knowledge in such a way that the stake holders of related domains are able to understand and value what is going on. This is where the uniform modeling potential of our approach may become useful. For, in that case the different stake holders get acquainted with the form in which knowledge is conceptualized when their knowledge processes are modeled. This familiarity with the form comes in handy when stake holders of different domains partake in Information System (IS) building. It is here that our process model for systematic conceptualization fits in. Its main characters are:

1) It reduces the continuous stream of reality to a finite amount of types of interpretation moments.

2) It conceptualizes hierarchically, not just by means of collections. The hierarchy is not dictated by a 
previously accepted ontology, but arises naturally in the course of the analysis.

3) Its goal orientedness provides means to ensure terminological consistency by keeping track of the processes in which common terms figure.

Below we will present our process model for systematic conceptualization. Since the model is founded upon the processual semiotics of the American polymath Ch. S. Peirce (1839-1914) we will start in section 2 with a treatment of some basic notions: process (2.1) and sign and habit (2.2). This treatment is also the occasion to argue that our way of conceptualization offers organizations the opportunity to execute self control. The more technical part of the paper starts in section 3, in which we recapitulate our model, including its logical interpretation. An illustrative example is presented in section 4. Finally, in section 5 we will draw conclusions.

\section{How to control conceptualization?}

If we confine our scope just to ER-modeling, it is possible to say that ER-models are used to create an accurate reflection of the structure of part of the real world in a database. But already if we only modestly extend our scope and ask what did trigger the modeling of a domain, we are forced to a more elaborate answer. The first thing to ask is what we do when we model, is it capturing real world events in a reflection or is it a matter of modeling our organization's future interactions with the world? Even with a narrow view on ER-modeling the answer must be that, at the very least, it is not only a matter of representation. For, although ER developed in order to remove redundancy and keep data consistent, if we implement a (normalized) model, we affect the behavior of everybody who is going to work for some reason with the realized information system.

With the rapid integration of information systems across departments, the growing model of entities and relations became hard to read. Contact with the users' demands was restored by specifying separate business processes, followed by a de-normalization of the overall ER-model from the perspective of the needs of the different roles engaged in those processes.

But how to proceed if we do not have a normalized model and/or the domain to be modeled is extensive, hosting different perspectives, stated in terms that may be vague or that lead to contradictions, in an environment where differences of interest seriously hamper progress? Here controlled conceptualization may make a difference. In the remainder of this section we will deal with our background notions as a preparation for the presentation of the model with which we deem controlled conceptualization feasible.

\subsection{Processes and conceptualization}

The concept of process is central to our approach. This is not primarily so because conceptualization itself can be regarded a process or because it is part of an embracing process aiming at the realization of an IS. The primary reason for taking process as a central concept resides in the fact that all we feel, think or do, is only felt, thought or done as part of some process(es). From the point of view of meaning as actualized meaning, processes are the basic units of meaning, not terms or propositions. The latter generalize over different processes by dissociation from any particular process. This will be clear if we realize that disambiguation as a rule proceeds by providing a proper context, thus embedding the ambiguous sign in the process intended by the utterer. Stake holders will be inclined to think about their concepts from the perspective of the processes in which they are embedded. So, explicating processes is a quite natural starting point for conceptualization. Since processes figure so prominently in our approach some words about our Peirce inspired understanding of the concept of process is called for. Hulswit succinctly summarized the Peircean concept of process thus (Hulswit, 1999):

A process is "a continuous sequence of events that derives its unity or internal order (distinguishing it from other processes) from a final cause, which directs the sequence to some end state which itself may evolve."

Several notions in this definition deserve some elucidation.

A process is a continuous sequence of events:

a) Continuous because events in a process are not separate from each other.

b) Events because there are distinguishable parts of a process that can be articulated as facts expressed in propositions.

Unity or internal order is derived from a final cause:

a) The goal of the process, the desired end state, provides the Reason of the process.

b) Only what contributes to the realization of the goal ought to be part of the model that describes the process.

The final cause directs the sequence to some end state: An event starts up a goal oriented habit that governs the process towards its terminating event.

The end state itself may evolve during the process (recursively):

The terminating event needs not to be fixed beforehand in detail, it may be general or vague. 
In this section we looked at processes in a very general way, now we zoom in on the different kinds of aspects that we must distinguish in all the kinds of sign processes, including those pertinent to IS.

\subsection{Signs, habits and conceptualization}

Peirce did hold from very early on that we have no power to think without signs (cf. CP 5.265). ${ }^{1}$ But if every thought always partakes in a sign, then the flow of thought, as far as it is connected and influential, is representable by an argument. So, it need not wonder us that throughout his life he kept refining his thought on signs, leaving us a tremendous corpus devoted to the study of signs in many of its aspects. This most certainly is not the place to trace the development of that thought or to lay bare the ramifications of the assumption. In the space at our disposal we will not even have the possibility to deal with the bare essentials of the core of his sign theory, but at the same time we will have to present an advancement of the basic theory which comes down to a transfer of the concept of 'sheet' from his logic to his semiotics. We start with the later and will afterwards introduce the relevant terminology 'on the fly'.

In analogy with the 'Sheet of Assertion' $\left(S_{A}\right)$ Pierce introduced in the context of his logic (cf. CP 4.396), we introduce a 'Semiotic Sheet' $\left(S_{S}\right)$ (van Breemen and Sarbo, 2007). The difference between the two is that the $S_{A}$ represents what is taken for granted to be true in the Universe of Discourse by all participants in an exchange of ideas, while the $S_{S}$ represents all that is believed by a group of participants or by an individual. Let us assume that the $S_{S}$ has at least two modalities. The actual $\mathrm{S}_{S}$ holds what at a given moment actually is believed, it is the state the $S_{S}$ is in at any given moment. The potential $S_{S}$ contains all beliefs that may become actualized some time.

Now, under the assumption that all thought is in signs, lets try to describe what happens if something demanding attention gets inscribed on a $S_{S}$ in the state it is actually in. See fig. 1 for a summarization of technical terms (left) and their approximate counterparts in mundane language (right). Although the 9 terms suggest that they are signs on their own, this is not the case. They are best regarded as analytical moments that surface if we are going to analyze goal oriented processes or reasoning.

The terms of fig. 1:

The qualisign position indistinctly involves all that is needed to reach a conclusion. Analytically it involves what is offering itself for interpretation (the

\footnotetext{
${ }^{1} \mathrm{~A}$ reference to (Peirce, 1931) is given by volume and paragraph, separated by a point.
}
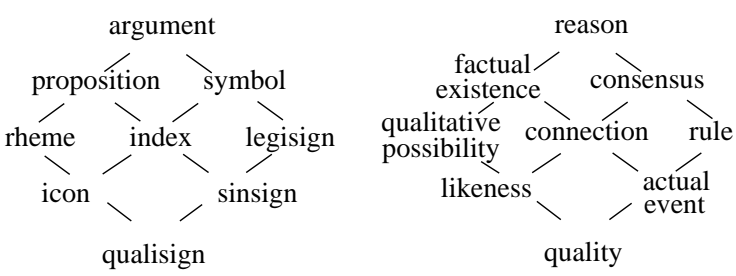

Figure 1: Sign aspects and corresponding mundane terms

effect), the actual state the $S_{S}$ is in and all that may be contributed by the $S_{S}$ written upon in the process of interpretation. State and effect appear as indistinct qualities.

The icon position discriminates the state and effect according to their form, but without any interpretation. It is the single occurrence of a potential likeness. Looked at it from a bottom up perspective the process can go on in many directions, none of which is recognized at this point.

The sinsign ${ }^{2}$ position indicates the co-occurrence of the state and effect on a particular $S_{S}$ as an actual event.

The index position indicates the actual connection of all moments involved in the interpretive process, by this it expresses the permanence of a process on a $S_{S}$. At the same time it expresses the latent connection between what is actualized in this process and what is potentially contained in this actualized $S_{S}$.

The legisign generalizes the occurrence (sinsign) of the instances of a form (icon). In learning this is a bottom up process, but if the form is known the actual event triggers a rule-like habit that classes all the instances of that form with a type. A legisign only surfaces by means of its instances, but as a habit it has a real influence.

The rheme position indicates the moment the form (icon) in its here and now actuality (sinsign) addresses the $S_{S}$ in order to retrieve all possible ways in which to deal with it, to contextualize it, but only potentially so. Creativity heavily draws upon this moment: figuring out how to possibly deal with the input (qualitative possibility).

The symbol position indicates the moment a familiar sign (legisign, involving a sinsign-icon) gets connected (index) with a habit of interpretation that connects the sign implied in the effect with its object. Object here used as in "What is the object of your remark?" The stronger this convention, the harder it is to be creative. At this point however the habit is still not operational.

The dicent indicates the position in which a specific

\footnotetext{
${ }^{2}$ 'Sin' as in 'singular'.
} 
possibility is asserted for actual use in the argument in a particular case. Out of all possible interpretations one is actually asserted (factual existence).

The argument position indicates at least two moments. On the one hand it indicates the whole process leading to the conclusion/response as its reason, on the other it states the conclusion/response, ready to enter a subsequent process.

What does this approach offer to conceptualization? In order to establish that it is useful to introduce a third modality of the $S_{S}$, the modality of law or operational habit. What is inscribed on a $S_{S}$ in this modality has real influence and as such fulfills the essential function of a sign, according to Peirce:

"It appears to me that the essential function of a sign is to render inefficient relations efficient, - not to set them into action, but to establish a habit or general rule whereby they will act on occasion" (CP 8.332).

But isn't that the kind of sign we want to develop if we are building an IS, at a level as much integrated as possible? In order to realize that, we regard the different stake holders as Semiotic Sheets that contain the knowledge we need. When we analyze we write the results on different sheets, for each process to be modeled one. We do this by combining these sheets until all that is needed for the processes is made explicit. We merge and sequence the different sheets on a sheet in which the common goals are regulative. Practical constraints, conflicts of interest and ambiguities will arise in the process. But they have a reason and can be discussed in an understandable way, since they can be connected to what is going on. That offers prospects for resolution. The question we pose may be summarized thus: Is it possible to develop a robust strategy for conceptualization by taking a Peircean semiotic account of cognitive activity as a starting point?

\section{The model}

In past research we suggested that Peirce's nine types of meaning aspects are generated in each sign recognition process (Sarbo, 2007). In this section we restrict ourselves to a brief recapitulation of the basic ideas.

The appearing sign (external effect) is interacting with the $S_{S}$ occurring in a state. According to our model, the recognition of this sign (phenomenon) can be established through the recognition of the relation between state $(A)$ and effect $(B)$, in the context of knowledge about earlier interactions $(\neg A, \neg B)$ possessed by the involved $S_{S}$. We assume that both state and effect are presented as input qualities appearing in a 'primordial soup' ([A B $]$ ). Sign recognition can be modeled by a processing schema, as shown in fig. 2 .

(1) sorting $([\mathrm{A}],[\mathrm{B}])$ : the identification of the two types of qualities in the 'primordial soup';

(2) abstraction (A, B): the separation of the collections of the two types of qualities;

(3) complementation $((A, \neg B),(B, \neg A))$ : the linking of the qualities with information from the context;

(4) predication $((A, \neg B)-(B, \neg A))$ : the establishment of a relation between the completed qualities.

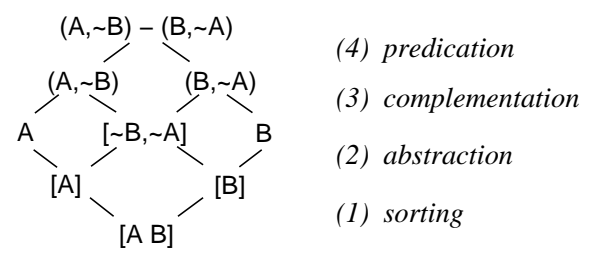

Figure 2: The processing schema of sign recognition

A logical analysis of our processing schema has been introduced in (Sarbo, 2007). The results of this analysis are recapitulated in fig. 3. An essential element of a logical interpretation is the abstraction of a common meaning of the two types of input qualities, which is the concept of a logical variable. Because we have two types of qualities, which are independent, the logical interpretation requires the introduction of two variables. These are denoted by $A$ (state) and $B$ (effect).

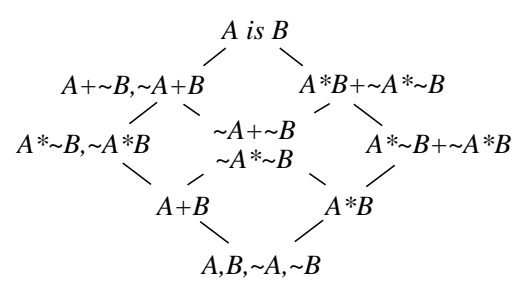

Figure 3: The logical interpretation of sign recognition

\section{An example}

There are two dimensions along which our theory of conceptualization can be explored. The first is related to its potential for merging knowledge (concepts) obtained by the different stake holders representing different views (Lankhorst et al., 2005). This can be illustrated with the specification of a common problem by a conceptual specialist and some domain 
expert(s). Full individual specifications, each expressing a certain point of view, are merged to a single meaningful relation, by unifying concepts of identical meaning aspects to a single sign, through coordination. The second way of exploration capitalizes on the potential of the theory to be applied recursively, which is beneficial for the conceptualization of complex phenomena. This section gives an illustration of such a problem. Let us emphasize that both types of exploration are a direct consequence of the uniform representation provided by the theory outlined in this paper.

\subsection{Conceptualization of a complex problem}

Assume our task is the generation of a meaningful representation of a company's strategy. From an abstract point of view such a strategy can be characterized by the company's reaction on supply and demand. According to the theory of this paper, such a reaction can be conceptualized as a relation between supply and demand where supply can be interpreted as the products produced by the company, which are available for some time (state), and demand as the suddenly appearing request dictated by the customers (effect).

The simultaneously appearing supply and demand (input qualities) define our company involved in a business event. The interpretation of the two types of qualities in themselves (individual analysis) provides us with a definition of $A$ and $B,{ }^{3}$ and through the interpretation of the relation between them (relational analysis) we may obtain a meaningful conceptualization of our company's reaction to the input event. That relation may also rely on complementary factors like the economic perspective taken, either profit maximization or purchasers satisfaction, which views in turn are intimately related to the more primitive concepts of price and selling events, that themselves can be specified as phenomena. The next section is an attempt to introduce a specification for selling events; an analogous treatment of the concept of price is left to the reader.

\subsection{Nested phenomenon: selling event}

A selling event is between a dealer and a customer. The specification below is given from the dealer's perspective; a description from the purchaser's point of view is possible, but it may be less general, as the pur-

\footnotetext{
${ }^{3}$ We refer to the status of a sign by means of the corresponding logical expression.
}

chaser's motivation for buying or not buying a product can be more difficult to set out.

We assume that a selling event, as a phenomenon, is defined by the purchaser's selection of certain products and services. The products can be interpreted as a state, in which, the services appear as an effect. For example, dealer and customer are having a conversation while they are looking at a collection of products, when suddenly the dealer recognizes (e.g. 'sees' in a saccade), the purchaser's selection of the services he or she wishes to be delivered with the products. Additionally we assume that a selling event may also be subject to complementary factors like the different forms of the transfer of ownership and the various judicial conditions a service may have to comply with.

The goal of this section is not the analysis of a concrete selling event, however. Our focus is on the interpretation of the relation between product and service, in general. Accordingly, we will assume that the input contains abstract qualities, which are: product $(A)$, service $(B)$, form of ownership $(\neg A)$ and judicial conditions $(\neg B)$. For example, $\neg A$ may refer to purchase, lease or rental, and $\neg B$ to copyright, knowhow and organizational conditions (e.g. a computer network, as a product, can be sold only as a whole, not in parts). The representation of abstract concepts, as qualities, is a non trivial question that we cannot discuss in detail, because of lack of space. The abstract concepts of selling are introduced as stages of the recognition process of an abstract selling event, as a sign (cf. fig. 2).

$[\mathrm{A}]=A+B$ : A product $(A)$ can be sold with a service $(B)$; or, a service $(B)$, for example, a know-how, can be available through a certain product $(A)$.

$[\mathrm{B}]=A * B:$ A product may provide a certain service only; or, a service can be available only in combination with a certain product (product-service dependency, as an actual event).

$[\neg \mathrm{A}, \neg \mathrm{B}]=\neg A+\neg B, \neg A * \neg B$ : The relation between the complementary factors of selling events like the various forms of ownership and the corresponding judicial issues $(\neg A+\neg B)$, and how those forms of ownership are regulated by law $(\neg A * \neg B)$.

$\mathrm{A}=A * \neg B, \neg A * B$ : Product specification like catalog information about products $(A * \neg B)$ and services $(\neg A * B)$, in general.

$\mathrm{B}=A * \neg B+\neg A * B$ : The reconciliation of purchaser's need for products and services, from the dealer's point of view. This is the concept of the habitual way of bargaining, which is a law-like relation or rule between products $(A * \neg B)$ and services $(\neg A * B)$ that can be sold in combination. This rule includes the possibility that products and services can be sold independently from each other (in conformity with 
the 'or' operation in the logical expression of $\mathrm{B})$. For example, one may buy $A$ without $B(A * \neg B)$ or, $B$ without $A(\neg A * B)$, or both. Notice that in this case the law-like relation identified as 'bargaining', a concept that most of us are familiar with, is automatically revealed by this conceptualization process (except its name, of course). This indicates that in cases in which we are not familiar with the concepts they are generated by the model.

$(\mathrm{A}, \neg \mathrm{B})=A+\neg B, \neg A+B$ : The interrelated product and service are actualized as constituents of a selling event. This includes the full specification of product and service according to the purchaser as well as the dealer, tacitly implicating their agreement (cf. bargaining in context).

$(\mathrm{B}, \neg \mathrm{A})=A * B+\neg A * \neg B$ : The characteristic property involved in any selling event, defined by a suitable combination of product and service $(A * B)$ and nothing else $(\neg A * \neg B)$ or, alternatively, the relation between the selected product and service $(A * B)$, in the light of their ownership forms and judicial conditions like the transfer of ownership through leasing or contract $(\neg A * \neg B)$.

$(\mathrm{A}, \neg \mathrm{B})-(\mathrm{B}, \neg \mathrm{A})=A$ is $B$ : The meaningful description of the input phenomenon, as a selling event.

\subsection{Main phenomenon continued}

The meaning of the nested phenomenon can be represented in the nesting phenomenon, degenerately, as a quality. As a result, the meaningful concept of a 'selling event' is reduced to complementary information $(\neg B)^{4}$ mediating supply and demand to their interpretation as the company's supply and the customers' demand and, finally, to a meaningful representation of our company's strategy. The context of price $(\neg A)$ and selling events $(\neg B)$, is an expression of their close interaction in a market mechanism $(\neg A * \neg B)$, but also of their possible influence as additional regulative factors, like environmental and political demand $(\neg A+\neg B)$.

The interpretation of the context along a continuum marked by 'liberal', on the one side, and 'state controlled', on the other, may provide a representation of our company (' $A$ is $B$ ' as a conclusion), as a more commercial profit oriented enterprise or a more non-profit organization, respectively.

\footnotetext{
${ }^{4}$ Now $\neg B$, but also $A, B$ and $\neg A$ refer to the qualities of the nesting phenomenon.
}

\section{Conclusions}

For systematic conceptualization of a domain, a process model is needed. This has several reasons. It minimizes unwanted ambiguity of the terms used by consistently extracting them from the processes in which they function. It enhances communication with and between stake holders about their domains by the use of concepts in context. And it facilitates strategic decision making in an early stage of IS building by enabling the re-thinking of the processes that make up any organization; after all goals can be reached in different ways.

The approach presented in this paper is closely related to cognitive activity interpreted as a sign process. The finite amount of types of meaningful interpretation moments (cf. fig. 1) facilitates the development of a common language between stake holders, as well as between stake holders and end-users. The order relation between the interpretation moments as well as between the processes increases the possibility to control conceptualization. The goal oriented nature of our processual model is well equipped to uncover hidden tensions by systematically keeping check of the relations between ends and means.

\section{REFERENCES}

Hulswit, M. (1999). From Cause to Causation : A Peircean Perspective, volume 90 of Philosophical Studies Series. Kluwer, Dordrecht.

Lankhorst, M., van der Torre, L., Proper, H., Arbab, F., and Steen, M. (2005). Viewpoints and visualisation. In Lankhorst, M., editor, Enterprise architecture at work: Modelling, Communication, and Analysis, pages 147-190, Berlin (Germany). Springe-Verlag.

Liu, K. (2000). Semiotics in Information Systems Engineering. Cambridge University Press, Cambridge (UK).

Peirce, C. (1931). Collected Papers of Charles Sanders Peirce. Harvard University Press, Cambridge.

Sarbo, J. (2007). On the logic underlying common sense. In Cardoso, J., Cordeiro, J., and Filipe, J., editors, ICEIS, pages 395-400, Funchal, Madeira (Portugal). INSTICC.

van Breemen, A. and Sarbo, J. (2007). Sign processes and the sheets of semeiosis $\left(\mathrm{S}_{S}\right)$. In Liu, K., editor, 10th International Conference on Complexity in Organisational and Technological Systems (ICOS), pages 89-98, Sheffield (UK). 